\title{
Low neutralizing antibody responses in WM, CLL and NHL patients after the first dose of the BNT162b2 and AZD1222 vaccine
}

\author{
Maria Gavriatopoulou ${ }^{1}$ (1) - Evangelos Terpos ${ }^{1}$ - Efstathios Kastritis ${ }^{1}$. Alexandros Briasoulis ${ }^{1}$. \\ Sentiljana Gumeni ${ }^{2} \cdot$ Ioannis Ntanasis-Stathopoulos ${ }^{1} \cdot$ Aimilia D. Sklirou $^{2} \cdot$ Panagiotis Malandrakis $^{1}$. \\ Evangelos Eleutherakis-Papaiakovou' ${ }^{1} \cdot$ Magdalini Migkou $^{1} \cdot$ loannis P. Trougakos ${ }^{2} \cdot$ Meletios A. Dimopoulos $^{1}$
}

Received: 20 May 2021 / Accepted: 12 July 2021 / Published online: 20 July 2021

(c) The Author(s), under exclusive licence to Springer Nature Switzerland AG 2021

\begin{abstract}
Vaccination against SARS-CoV-2 is considered as the most important preventive strategy against COVID-19, but its efficacy in patients with hematological malignancies is largely unknown. We investigated the development of neutralizing antibodies (NAbs) against SARS-CoV-2 in patients with Waldenstrom Macroglobulinemia (WM), Chronic Lymphocytic Leukemia (CLL) and Non-Hodgkin Lymphoma (NHL). After the first dose of the vaccine, on D22, WM/CLL/NHL patients had lower NAb titers compared to controls: the median NAb inhibition titer was 17\% (range 0-91\%, IQR 8-27\%) for WM/CLL/NHL patients versus 32\% (range 2-98\%, IQR 19-48\%) for controls $(P<0.001)$. Only $8(14 \%)$ patients versus $114(54 \%)$ controls developed NAb titers $\geq 30 \%$ on D22 $(p<0.001)$. Our data indicate that the first dose of both BNT162b2 and AZD1222 leads to lower production of NAbs against SARS-CoV-2 in patients with WM/CLL/NHL compared to controls of similar age and gender and without malignant disease. Even though the response rates were not optimal, vaccination is still considered essential and if possible should be performed before treatment initiation. These patients with suboptimal responses should be considered to be prioritized for booster doses.
\end{abstract}

Keywords SARS-CoV-2 · COVID-19 • Vaccine - Waldenstrom Macroglobulinemia Chronic Lymphocytic Leukemia Non-Hodgkin Lymphoma · Antibodies · BNT162b2 · AZD1222

Patients with lymphoproliferative disorders including those with Waldenström Macroglobulinemia (WM) and Chronic Lymphocytic Leukemia (CLL) are at increased risk of bacterial and viral infections and also at increased risk for severe disease and death from COVID-19 [1], due to their immunocompromised status, older age and comorbidities [2-4].

Due to defects in immune effector cells [5], associated with the B-cell disorder and the use of certain therapies, there may be a suboptimal immune response to vaccines. Vaccination against SARS-CoV-2 is considered as the most

Maria Gavriatopoulou

mariagabria@gmail.com

1 Department of Clinical Therapeutics, School of Medicine, Alexandra General Hospital, National and Kapodistrian University of Athens, 80 Vas. Sofias Avenue, 11528 Athens, Greece

2 Department of Cell Biology and Biophysics, Faculty of Biology, National and Kapodistrian University of Athens, Athens, Greece important preventive strategy against COVID-19, but its efficacy in patients with hematological malignancies is largely unknown [6]. The BNT162b2 mRNA and the AZD1222 viral vector vaccines against SARS-CoV-2 have shown significant efficacy in healthy adults $[7,8]$. The first BNT162b2 dose confers some protection among nursing facility members [9, 10], health workers and octogenarians [11]. However, among 167 patients with CLL, only $39.5 \%$ had a positive antibody-mediated response to the BNT162b2 vaccine [12]. Low antibody responses have been also shown among 48 elderly myeloma patients who received the first dose of BNT162b2 vaccine [13]. However, in contrast to CLL and myeloma, patients with myeloproliferative neoplasms present an antibody response to BNT162b2, which is similar to that of the general population [14-16]. In this context, we report the development of neutralizing antibodies (NAbs) against SARS-CoV-2 in patients with WM, CLL and other NHLs after the first dose of the BNT162b2 and AZD1222 vaccines. 
Major inclusion criteria for the study included: (i) age above 18 years; (ii) presence of asymptomatic or active WM, CLL, or other low-grade NHL irrespective of the treatment phase; and (iii) eligibility for vaccination. Volunteer controls of similar age and gender were also included in this analysis. Major exclusion criteria for both patients and controls included the presence of: (i) autoimmune disorders or active malignant disease; (ii) HIV or active hepatitis B and $\mathrm{C}$ infection and (iii) end-stage renal disease. The patients and controls enrolled participate in a large prospective study (NCT04743388) for the kinetics of anti-SARS-CoV-2 antibodies after COVID-19 vaccination in healthy subjects and patients with hematological malignancies or solid tumors.

After vein puncture, the serum of both patients and controls was collected on day 1 (D1; before the first BNT162b2 or AZD1222 dose) and on day 22 (D22; before the second dose of the BNT162b2 or 3 weeks post the first AZD1222 dose). Serum was separated within $4 \mathrm{~h}$ from blood collection and stored at $-80{ }^{\circ} \mathrm{C}$ until the day of measurement. NAbs against SARS-CoV-2 were measured using FDA approved methodology (ELISA, cPass ${ }^{\mathrm{TM}}$ SARS-CoV-2 NAbs Detection Kit; GenScript, Piscataway, NJ, USA) [17] on the above-mentioned timepoints. Samples of the same patient or control were measured in the same ELISA plate. The study was approved by the respective Ethical Committees in accordance with the Declaration of Helsinki and the International Conference on Harmonization for Good Clinical Practice. All patients and controls provided written informed consent prior enrollment in the study.

Study population included 58 patients ( 28 males/30 females; median age: 75 years, range: $40-88$ years, IQR: 63-81 years) and 213 controls (100 males/113 females; median age: 75 years, range: $61-95$ years, IQR: 63-83 years), who were vaccinated during the same period, at the same vaccination center (Alexandra Hospital, Athens, Greece). The patients were vaccinated based on the Greek vaccination program that prioritizes patients with malignancies for COVID-19 immunization. Forty-four patients (76\%) were vaccinated with the BNT162b2 and 14 (24\%) with the AZD1222 vaccine. Among the control group the proportion was similar with the patient group; $149(70 \%)$ were vaccinated with the BNT162b2 and 64 (30\%) with the AZD1222 vaccine $(p=0.38)$.

The characteristics of the patients are depicted in Table 1. At the time of vaccination, 17 (46\%) out of 37 symptomatic patients were receiving therapy, $19(51 \%)$ were in remission after prior treatment and did not receive any therapy at the
Table 1 Characteristics of patients with WM/CLL/NHL

\begin{tabular}{ll}
\hline Number of patients (Male/Female) & 58 (28/30) \\
Age in years, median (range) & 75 (range 40-88, IQR:63-81) \\
Symptomatic disease $n=37$ & WM: $n=17$ \\
& CLL: $n=12$ \\
& NHL: $n=8$ \\
Asymptomatic disease $n=21$ & WM: $n=11$ \\
& CLL: $n=10$ \\
Symptomatic disease vaccinated with BNT162b2 $n=29$ & WM: $n=12$ \\
& CLL: $n=11$ \\
Symptomatic disease vaccinated with AZD1222 $n=8$ & NHL: $n=6$ \\
& WM: $n=5$ \\
Asymptomatic disease vaccinated with BNT162b2 $n=15$ & CLL: $n=1$ \\
& NHL: $n=2$ \\
Asymptomatic disease vaccinated with AZD1222 $n=6$ & WM: $n=5$ \\
Patients on active treatment at the time of vaccination & CLL: $n=10$ \\
& WM: $n=6$ \\
& $n=17$ \\
Type of therapy & WM $n=5$ \\
WM & CLL $n=8$ \\
CLL & NHL $n=4$ \\
NHL & \\
& Rituximab-Ibrutinib $n=3$ \\
& Bortezomib-Rituximab-Ibrutinib $n=2$ \\
& Venetoclax $n=1$ \\
& Ibrutinib $n=7$ \\
& Rituximab $n=2$ \\
& Bendamustine-Rituximab $n=2$ \\
&
\end{tabular}

WM Waldenström Macroglobulinemia, CLL Chronic Lymphocytic Leukemia, NHL Non-Hodgkin Lymphoma 
Fig. 1 Kinetics of neutralizing antibodies in WM/CLL/ NHL patients and age-matched controls after vaccination with the first dose of the BNT162b2 mRNA and AZD1222 vaccine. On D22, patients had lower production of NAb inhibition titers compared to controls of similar age and gender (see text). Only 3 patients had NAb titers of equal or more than $50 \%$

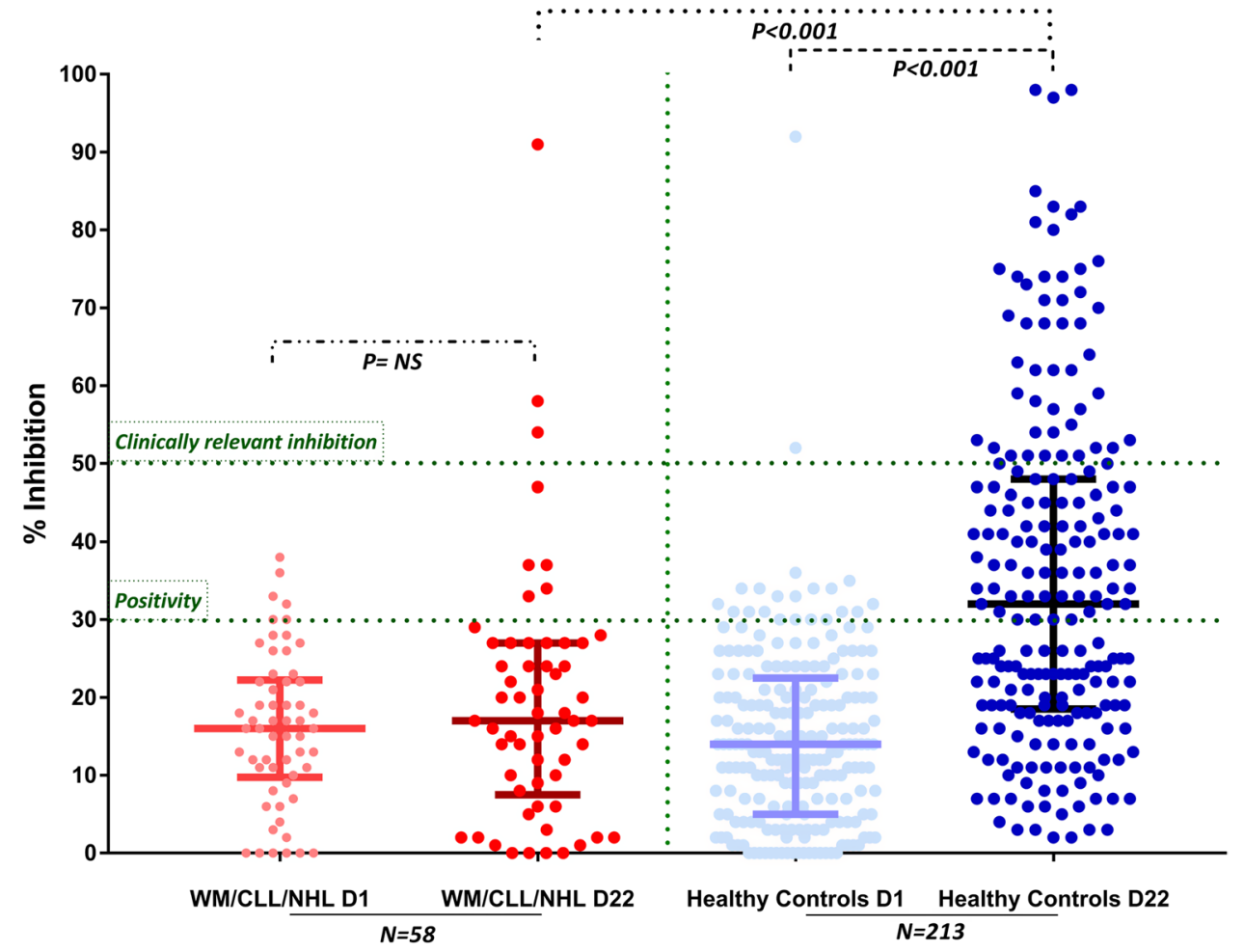

time of vaccination and 21 (36\%) out of 58 patients had asymptomatic disease.

On D1, $6(10.3 \%)$ patients and $21(9.9 \%)$ controls had NAb titers of $\geq 30 \%$ (positivity cut-off); there was no difference regarding the NAb titers between patients and controls on D1 $(p=0.9)$. None of them had a prior history of known COVID-19. After the first dose of the vaccine, on D22, WM/CLL/NHL patients had lower NAb titers compared to controls: the median NAb inhibition titer was 17\% (range 0-91\%, IQR:8-27\%) for WM/CLL/ NHL patients versus 32\% (range 2-98\%, IQR:19-48\%) for controls; $P<0.001$ (Fig. 1). More, specifically, only 8 (14\%) patients versus 114 (54\%) controls developed NAb titers $\geq 30 \%$ on D22 $(p<0.001)$. The respective number of patients and controls who developed NAb titers $\geq 50 \%$ (clinically relevant viral inhibition [18]) was $3(5 \%)$ and $50(24 \%)$, respectively $(p=0.002)$. Out of these 3 patients, 2 had symptomatic WM and 1 asymptomatic CLL. The WM patients were in remission, without receiving any therapy ( 1 in complete remission and 1 in partial remission) both after treatment with anti-CD20 based therapeutic regimens (dexamethasone-cyclophosphamiderituximab; DRC). Both patients had the uninvolved immunoglobulins after treatment within normal limits and were off treatment for more than 12 months. The remaining 5 patients that developed positivity $\geq 30 \%$ were all WM (4 asymptomatic/1 symptomatic). The symptomatic patient was in remission, out of treatment for more that 12 months and had been previously treated with DRC. The univolved immunoglobulins were within normal ranges for all 5 patients. Among the symptomatic patients with low response rates $(<30 \%)(n=37), 17$ were on active treatment at the time of vaccination ( 7 with ibrutinib, 3 with ibrutinib-rituximab, 2 with ibrutinib-bortezomibrituximab, 1 with venetoclax, 2 with rituximab and 2 with bendamustine-rituximab).

Our data indicate that the first dose of both BNT162b2 and AZD1222 leads to lower production of NAbs against SARS-CoV-2 in patients with WM/CLL/NHL compared to controls of similar age and gender and without malignant disease. To our knowledge this is the first report for the effects of AZD1222 vaccine in patients with low grade lymphoproliferative neoplasms. The results were independent of the vaccine type. Therapeutic regimens that deplete B-cells may impair immune response to vaccines. Patients treated with ibrutinib, venetoclax and/or anti-CD20 antibodies were unlikely to respond to a single dose of vaccine and this is confirmed by our results. Ibrutinib blocks the B-cell receptor signaling, in both malignant and normal B-cells and therefore impairs the humoral response to vaccination. The exposure to B-cell depleting agents, including antiCD20 antibodies, reduces response to influenza vaccine, pneumococcal polysaccharide vaccine and other vaccines [19]. Furthermore, hypoglobulinemia might be associated with inferior antibody response among patients with CLL and COVID-19 [20]. Interestingly, it seems that patients who completed their treatment and remained in response at the time of vaccination were more likely to produce NAbs 
and this is probably related to a reconstitution of humoral immunity.

Our results also suggest that a second timely vaccine dose is necessary for patients with hematological malignancies that deregulate the immune homeostasis, and especially for the elderly [21]. A shorter time interval between the two doses of the AZD1222 would be relevant, as well [22]. Similarly, patients with solid cancer present a suboptimal humoral response following the first dose of the BNT162b2 and they should receive an early second dose (21 days after the first shot) [23-25]. Unfortunately, we did not have an adequate number of patients in order to perform statistically robust subgroup analysis according to type of therapy or presence/absence of hypogammaglobulinemia. Targeted therapies seem to negatively affect the NAb production (after a single dose), although higher patient numbers are required to evaluate the exact effect of each regimen on the immune responses of anti-SARS-CoV-2 vaccination.

In summary, the antibody-mediated response to SARS$\mathrm{CoV}-2$ vaccines in patients with WM/CLL/NHL is considerably affected by both the underlying disease and the treatment. Even though the response rates were not optimal, vaccination is still considered essential and if possible should be performed before treatment initiation. These patients with suboptimal responses should be considered to be prioritized for booster doses, potentially with a different vaccine type [26]. For these patients prolonged self-protection measures, such as mask wearing and social distancing, are necessary.

Acknowledgements We thank Ioanna Charitaki, $\mathrm{RN}^{1}$; Tina Bagratuni, $\mathrm{PhD}^{1}$; Christine Ivy Liacos, $\mathrm{PhD}^{1}$; Nikoletta-Aikaterini Kokkali, $\mathrm{RN}^{1}$; Nefeli Mavrianou-Koutsoukou, $\mathrm{PhD}^{1}$; Dimitrios Patseas, $\mathrm{PhD}^{1}$ and Mrs Stamatia Skourti ${ }^{1}$ for administrative, technical, or material support; Eleni-Dimitra Papanagnou, $\mathrm{PhD}^{2}$ for acquisition, analysis, or interpretation of data. We also thank SYN-ENOSIS (Greece), AEGEAS (Greece) and IEMBITHEK (Greece) for partially funding this study, as well as all of the study participants for donating their time and samples.

Author's contribution MG, ET and MAD designed research, performed research, analyzed data and wrote the paper; AB and IPT contributed vital new reagents or analytical tools, performed research, analyzed data, reviewed all paper drafts and gave approval to final version; EK, SG, IN-S, ADS, PM, E-EP, and MM performed research, analyzed data, reviewed all paper drafts and gave approval to final version.

Data availability Raw data are available upon reasonable request from the corresponding author.

\section{Declarations}

Conflict of interest The authors declare no relevant conflict of interest.

Ethics approval The study was conducted according to the guidelines of the Declaration of Helsinki and approved by the Ethics Committee of "Alexandra" General Hospital, Athens, Greece (protocol code 900, 24 December 2020).

\section{References}

1. Huang C, Wang Y, Li X, et al. Clinical features of patients infected with 2019 novel coronavirus in Wuhan. China Lancet. 2020;395:497-506.

2. Tadmor T, Welslau M, Hus I. A review of the infection pathogenesis and prophylaxis recommendations in patients with chronic lymphocytic leukemia. Expert Rev Hematol. 2018;11:57-70.

3. Mato AR, Roeker LE, Lamanna N, et al. Outcomes of COVID19 in patients with CLL: a multicenter international experience. Blood. 2020;136:1134-43.

4. Scarfo L, Chatzikonstantinou T, Rigolin GM, et al. COVID-19 severity and mortality in patients with chronic lymphocytic leukemia: a joint study by ERIC, the European research initiative on CLL, and CLL Campus. Leukemia. 2020;34:2354-63.

5. Ravandi F, O’Brien S. Immune defects in patients with chronic lymphocytic leukemia. Cancer Immunol Immunother. 2006;55:197-209.

6. Gavriatopoulou M, Ntanasis-Stathopoulos I, Korompoki E, et al. SARS-CoV-2 vaccines in patients with multiple myeloma. Hemasphere. 2021;5:e547.

7. Polack FP, Thomas SJ, Kitchin N, et al. Safety and efficacy of the BNT162b2 mRNA Covid-19 vaccine. N Engl J Med. 2020;383:2603-15.

8. Emary KRW, Golubchik T, Aley PK, et al. Efficacy of ChAdOx1 nCoV-19 (AZD1222) vaccine against SARS-CoV-2 variant of concern 202012/01 (B.1.1.7) an exploratory analysis of a randomised controlled trial. Lancet. 2021;397:1351-62.

9. Gharpure R, Patel A, Link-Gelles R. First-dose COVID-19 vaccination coverage among skilled nursing facility residents and staff. JAMA 2021;325(16):1670-1.

10. Saadat S, Tehrani ZR, Logue $\mathrm{J}$ et al. Binding and neutralization antibody titers after a single vaccine dose in health care workers previously infected with SARS-CoV-2. JAMA 2021;325(14):1467-9.

11. Terpos E, Trougakos IP, Apostolakou F et al. Age- and genderdependent antibody responses against SARS-CoV-2 in health workers and octogenarians after vaccination with the BNT162b2 mRNA vaccine. Am J Hematol 2021;96(7):E257-9.

12. Herishanu Y, Avivi I, Aharon A et al. Efficacy of the BNT162b2 mRNA COVID-19 vaccine in patients with chronic lymphocytic leukemia. Blood 2021;137(23):3165-73.

13. Terpos E, Trougakos IP, Gavriatopoulou M et al. Low neutralizing antibody responses against SARS-CoV-2 in elderly myeloma patients after the first BNT162b2 vaccine dose. Blood 2021;137(26):3674-6.

14. Harrington P, de Lavallade H, Doores KJ et al. Single dose of BNT162b2 mRNA vaccine against SARS-CoV-2 induces high frequency of neutralising antibody and polyfunctional T-cell responses in patients with myeloproliferative neoplasms. Leukemia 2021;1-5. https://doi.org/10.1038/s41375-021-01300-7.

15. Roeker LE, Knorr DA, Thompson MC et al. COVID-19 vaccine efficacy in patients with chronic lymphocytic leukemia. Leukemia 2021;1-3. https://doi.org/10.1038/s41375-021-01270-w.

16. Pimpinelli F, Marchesi F, Piaggio G, et al. Fifth-week immunogenicity and safety of anti-SARS-CoV-2 BNT162b2 vaccine in patients with multiple myeloma and myeloproliferative malignancies on active treatment: preliminary data from a single institution. J Hematol Oncol. 2021;14:81. 
17. Tan CW, Chia WN, Qin X, et al. A SARS-CoV-2 surrogate virus neutralization test based on antibody-mediated blockage of ACE2-spike protein-protein interaction. Nat Biotechnol. 2020;38:1073-8.

18. Walsh EE, Frenck RW Jr, Falsey AR, et al. Safety and immunogenicity of two RNA-based Covid-19 vaccine candidates. N Engl J Med. 2020;383:2439-50.

19. Yri OE, Torfoss D, Hungnes O, et al. Rituximab blocks protective serologic response to influenza A (H1N1) 2009 vaccination in lymphoma patients during or within 6 months after treatment. Blood. 2011;118:6769-71.

20. Roeker LE, Knorr DA, Pessin MS, et al. Anti-SARS-CoV-2 antibody response in patients with chronic lymphocytic leukemia. Leukemia. 2020;34:3047-9.

21. Collier DA, Ferreira I, Kotagiri P et al. Age-related immune response heterogeneity to SARS-CoV-2 vaccine BNT162b2. Nature 2021. https://doi.org/10.1038/s41586-021-03739-1.

22. Terpos E, Trougakos IP, Karalis V et al. Comparison of neutralizing antibody responses against SARS-CoV-2 in healthy volunteers who received the BNT162b2 mRNA or the AZD1222 vaccine: Should the second AZD1222 vaccine dose be given earlier? Am J Hematol 2021. https://doi.org/10.1002/ajh.26248.
23. Monin L, Laing AG, Munoz-Ruiz M, et al. Safety and immunogenicity of one versus two doses of the COVID-19 vaccine BNT162b2 for patients with cancer: interim analysis of a prospective observational study. Lancet Oncol. 2021;22:765-78.

24. Palich R, Veyri M, Marot $S$ et al. Weak immunogenicity after a single dose of SARS-CoV-2 mRNA vaccine in treated cancer patients. Ann Oncol 2021;32(8):1051-3.

25. Waissengrin B, Agbarya A, Safadi E, et al. Short-term safety of the BNT162b2 mRNA COVID-19 vaccine in patients with cancer treated with immune checkpoint inhibitors. Lancet Oncol. 2021;22:581-3.

26. Borobia AM, Carcas AJ, Perez-Olmeda M et al. Immunogenicity and reactogenicity of BNT162b2 booster in ChAdOx1-S-primed participants (CombiVacS): a multicentre, open-label, randomised, controlled, phase 2 trial. Lancet 2021;398(10295):121-30.

Publisher's Note Springer Nature remains neutral with regard to jurisdictional claims in published maps and institutional affiliations. 